\title{
基于智能网联的特种设备智能制造发展思路分析
}

\author{
刘三汇，陈祖志，石坤，李光海 \\ （中国特种设备检测研究院，北京 100029）
}

\begin{abstract}
摘要: 特种设备是国民经济建设和社会生活的重要基础设施, 在特种设备领域发展智能制造、实现特种设备制造业的转型升 级, 是 “中国制造 2025 ” 的重要组成部分。本文分析了特种设备的特点及发展现状, 梳理了发展特种设备智能制造的需求情况, 提出了推动特种设备制造业由大变强的有效手段及发展思路。研究提出, 发展特种设备智能制造的指导思想是采用并联式发 展道路, 并行推进特种设备行业工业 2.0、工业 3.0、工业 4.0 的发展; 发展重点是产品可追溯、质量管理智能化、动态风险 实时监控。在此基础上, 引入智能网联技术思路作为特种设备智能制造的发展路径, 分析智能网联的技术内涵, 着重从特种 设备智联网构建、智能应用开发两方面提出了基于该路径的特种设备智能制造关键任务, 以期为特种设备领域推进智能制造 提供理论参考。
\end{abstract}

关键词: 特种设备; 智能制造; 智能网联; 并联式发展

中图分类号: X933 文献标识码: A

\section{Intelligent Manufacturing of Special Equipment Based on Intelligent Connection}

\author{
Liu Sanjiang, Chen Zuzhi, Shi Kun, Li Guanghai
}

(China Special Equipment Inspection and Research Institute, Beijing 100029, China)

\begin{abstract}
Special equipment are important for the economic and societal development of a country. Implementing intelligent manufacturing in the special equipment field could facilitate the transformation and upgrades of the special equipment manufacturing industry of China, and it is also an essential component for the China Manufacturing 2025 plan. In this article, we first analyze the characteristics and current status of special equipment in China and summarize the demand for intelligent manufacturing of special equipment. Then we propose some measures and development ideas for promoting the special equipment manufacturing industry from big to strong. We propose that the guiding ideology should be parallel development; the Industry 2.0, Industry 3.0, and Industry 4.0 should be promoted in parallel in the special equipment industry. The development of intelligent manufacturing of special equipment should focus on product traceability, intelligent quality management, and real-time monitoring of dynamic risks. Based on this, intelligent connection is introduced as a technology path for developing intelligent manufacturing of special equipment, and the concept of intelligent connection is further analyzed. Furthermore, the key tasks for future development are building an intelligent connection network of special equipment and developing intelligent applications.
\end{abstract}

Keywords: special equipment; intelligent manufacturing; intelligent connection; parallel development

收稿日期 : 2019-12-26; 修回日期 : 2020-11-10

通讯作者: 刘三江, 中国特种设备检测研究院高级工程师、院长, 研究方向为特种设备安全管理及标准化工作;

E-mail: liusanjiang@csei.org.cn

资助项目：国家重点研发计划项目 “典型移动式承压类特种设备动态风险监管关键技术研究” (2017YFC0805605)

本刊网址：www.engineering.org.cn/ch/journal/sscae 


\section{一、前言}

我国已经成为制造大国，在全球制造业中 的占比约为 $20 \%$, 但还不是制造强国 [1]。为进 一步推动制造业由大变强, 我国实施 “中国制造 2025》”[2], 以信息化与工业化深度融合为主线, 以 “创新驱动、质量为先、绿色发展、结构优化、 人才为本” 为基本方针, 对于我国应对第四次科技 革命带来的新一轮国际竞争意义重大。智能制造是 我国制造业高质量发展的主攻方向、制高点、突破 口，也是德国工业 4.0、美国工业互联网等产业规 划的发展方向。目前, 我国对智能制造尚无确切定 义, 比较共性的认识是主要包括产品/装备智能化、 制造过程智能化、生产方式/产业模式新型化三方 面内容，有机结合、互为支撑、逐步推进，共同 构成智能制造系统 [1 4]。我国智能制造整体上仍 处于不断向深度与广度发展的初级阶段, 由于缺 乏具体的技术路径指引, 多数方向的推进速度都 较慢 [3]。

特种设备是国民经济建设和居民生活的重要基 础设施, 在经济社会发展中发挥着重要作用, 在特 种设备领域发展智能制造、实现特种设备制造业的 转型升级，是 “中国制造 2025 ” 的重要组成部分。 特种设备领域积极推进信息化工作，引导和推动 “互联网 + 特种设备” 的发展。2015 年, 国务院办 公厅印发《关于加快推进重要产品追溯体系建设的 意见》, 要求各地开展气瓶、电梯等安全质量追溯 体系建设; 2016 年, 国家市场监督管理总局提出 “互 联网 + 质检” 行动计划。就特种设备智能制造而言, 目前在理论、技术、法规、标准等方面的研究还基 本处于空白状态, 需要加紧对传统制造向智能制造 发展的影响因素、发展途径、解决方案以及智能制 造的商业模式、法律法规等开展研究, 以支撑特种 设备智能制造的高质量发展，其中最为紧迫的是选 择确定合理可行的技术途径。

已有研究 [5 9] 提出, 要在特种设备行业充分 应用大数据、物联网、人工智能 $(\mathrm{AI})$ 等技术, 构 建智能网联特种设备的发展思路, 分析智能网联特 种设备的科技与标准化需求以及检验、监管模式等。 基于此, 本文将智能网联理念引入特种设备制造环 节, 以之作为实现特种设备智能制造的技术途径并 论述具体的发展思路。

\section{二、特种设备发展现状及需求分析}

\section{（一）特种设备特点及发展现状}

1. 特种设备的技术与管理特点

《中华人民共和国特种设备安全法》规定, 锅 炉、压力容器（含气瓶）、压力管道、电梯、起重 机械、客运索道、大型游乐设施、场 (厂) 内专用 机动车辆共八大类别设备需按特种设备进行管理, 每一类又分为若干品种, 一些品种还进一步细分为 多个层级的子类。特种设备一旦失效, 对人身和财 产安全构成较大危险性, 为此国家专门设立政府部 门对其生产、经营、使用实施分类和全过程的安全 监督管理。特种设备普遍具有技术复杂度高、设计 要素多、生产过程复杂、使用环节多、操作要求高、 运维难度大、服役周期长等特点, 有效保障特种设 备产品质量及使用安全、避免事故，是一项富有挑 战性的工作, 也是一项系统工程。因此, 需要生产 者、经营与使用者、政府机构、第三方技术机构等 多方参与, 在特种设备的设计、制造、使用直至报 废的产品全生命周期中，加强科技、管理、人才、 经济等方面的持续投入。我国建立了基于传统技术 的特种设备质量与安全保障体系以及相应的法规标 准体系, 这套体系具有开放性, 能够不断吸收科技 创新成果进行优化调整。然而, 特种设备产品仍以 机械化、半自动化为主, 很多环节还是人工手动操 作的方式; 各环节应接受政府的强制性监管, 如设 计需要通过第三方机构审核鉴定，制造工艺需要经 过评定认可, 产品质量检验需要接受第三方监督, 使用过程需要由第三方机构定期进行安全可靠性再 评价，相关机构、人员须取得对应的资质与资格。

\section{2. 特种设备发展趋势}

特种设备的发展与工业、科技、经济、社会 的发展相互影响、相互促进。改革开放 40 多年 来, 随着经济发展和社会进步, 特种设备品种逐渐 增多、数量持续快速增长。截至 2019 年年底, 特 种设备总量已达 $1.525 \times 10^{7}$ 台（套）, 年增长率超 过 $11 \%$; 另有气瓶约 $1.64 \times 10^{8}$ 只、压力管道总长度

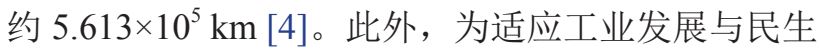
需求, 特种设备的结构更趋复杂, 介质、工况、环 境越来越苛刻, 设计载荷、压力、尺寸、速度等参 数不断提高且有些已接近极端化, 而运行、维护、 检验、服役周期也越来越长，导致质量、安全的影 
响因素更加多元化、耦合化。特种设备安全风险防 控的要素也越来越多、风险越来越高。

\section{3. 特种设备发展面临的挑战}

现有的特种设备质量、安全保障体系在提升特 种设备产品质量安全水平、降低事故率方面发挥了 重要作用, 但普遍存在技术含量不高、要素过多、 流程复杂、机制僵化、效率低下等问题，造成特种 设备发展的瓶颈性环节日益凸显, 尤其是产品质量 稳定性、安全保障可靠性、安全监管有效性等叒待 提升。当今世界正处在第四次科技革命的关口期, 新一轮国际竞争日趋激烈，科技创新密集活跃、 高速发展, 产业和社会变革日新月异, 立足科技 创新来推动特种设备产业的转型升级、提升质量 安全水平势在必行。

\section{（二）特种设备智能制造需求分析}

\section{1. 特种设备发展的新要求}

随着全球工业、科技、经济、社会发展新态势 的不断涌现, 加之特种设备自身发展出现新趋势, 对特种设备提出了一系列新的要求。(1)需要杜绝重 特大事故, 中央提出了 “坚决遏制重特大安全生产 事故发生” 的要求，而近几年特种设备相关重特大 事故仍时有发生, 严重影响经济发展和社会稳定。 (2)需要进一步提升总体安全水平，相关水平仍落后 于发达国家，部分设备万台死亡率仍为发达国家的 2 3 倍, 特种设备的安全状况相比国家和人民的要 求仍有较大差距。(3)需要进一步提升产品质量和安 全性能, 相关产品竞争力还较弱, 很多高端产品依 赖进口, 而出口产品主要是低技术含量、低附加值 产品且主要出口到欠发达国家和地区, 与建设质量 强国的发展目标存在差距。(4)需要提高安全监管的 效能, 行业安全监管人员数量不足与设备数量快速 增长之间的 “人机矛盾” 异常突出且长期得不到有 效解决, 安全监管业务压力大、风险高。(5)需要在 各环节构建新的业务模式, 数字经济的发展带来了 社会生产关系的变革，现有的政府监管及第三方 技术服务的业务模式、服务方式已不能满足市场 需求。

2. 特种设备智能制造的发展需求

第四次科技革命是以物联网、先进传感、AI、 大数据、云计算等现代信息技术为代表的新一轮科 技变革，紧抓第四次科技革命的发展契机，充分利
用现代信息技术，大力发展智能制造，是解决特种 设备领域当前面临的应用难题和发展瓶颈的有效途 径, 也是必由之路。

发展智能制造, 打造智能化特种设备产品, 是 提升特种设备产品竞争力的需求。研制智能电梯、 智能游乐设施、智能气瓶等产品, 开发个性化设计、 在线健康监测、实时状态诊断、缺陷精准检测、超 前感知预警、远程预防维护、自主状态调整、及时 高效应急等功能。在产品设计、安全状态识别、定 期检验、危险预警预报、状态控制调整、事故应急 响应等链条中的诸多方面实现智能化，优化产品设 计, 升级产品功能, 提升产品质量和安全性能。

发展智能制造, 实现特种设备数字化、智能化 生产, 是保证特种设备产品质量安全性能稳定可靠 的需求。在制造加工环节, 发展数字化车间、智能 工厂，采用智能化工艺装备与少人化甚至无人化、 连续化、自动化、智能化生产线; 采用精准化的产 品检测、质量检验分析技术，提高工艺的稳定性、 可控性、可调性以及参数精度的可靠性, 确保质量 检验的高效性及缺陷检出的精准性, 有效避免不合 格产品出厂。

发展智能制造, 构建特种设备智能化、智慧化 服务模式，是提高特种设备服务效能、实现风险精 准防控的需求。针对第三方技术服务、政府监管等 业务, 创建智能化、智慧化模式, 以数据为驱动、 以风险为准则, 以信息化、网络化、自动化、标准 化为基础, 以全生命周期全链条大数据为平台, 实 现自主感知、学习、分析以及信息共享、快速反应、 精准施策等功能, 提高服务能效、缓解人机矛盾。 发展智能制造, 最终实现安全风险的精准防控, 有 效避免事故的发生, 提高特种设备总体安全水平; 将设备、人员、环境、机构之间联网互动，有效降 低设备的系统风险, 避免重特大事故的发生。

\section{三、发展特种设备智能制造的策略与要点 分析}

\section{（一）发展特种设备智能制造的策略}

当前，国内不同类别、不同品种特种设备之间 以及不同企业之间的制造水平发展很不平衡, 有些 行业已经在推进智能制造并取得一定成绩, 如电梯、 大型游乐设施等行业的部分企业; 有些则刚刚起 
步，如管道、气瓶行业的部分企业; 有些则尚未起 步。因此, 特种设备推进智能制造的策略可确定为: 走工业 2.0 补课、工业 3.0 普及、工业 4.0 示范的并 联式发展道路 [10], 并行推进工业 2.0、工业 3.0、 工业 4.0 的发展。具体来看, 对于压力容器、工业 锅炉等承压类特种设备的制造，大多数企业目前基 本处于工业 1.0 时代, 大多数制造工作还需通过人 工或简单机械来完成, 如焊接、无损检测、宏观检 查等, 因此需要同时推进自动化、信息化、网络化 的发展; 电梯、大型游乐设施行业以及起重机械等 部分机电类特种设备, 其产品已经基本实现自动化, 部分制造企业的自动化、信息化已经发展到一定水 平, 可以着重推进智能制造的发展。

特种设备具有较大危险性, 保障其使用安全 性在任何时期都应放在首位并予以高度重视。特种 设备的智能化, 不仅不能以牺牲安全性为代价, 还 应以促进安全性的进一步提升为根本追求。特种设 备发展智能制造, 须考虑所采用信息技术的信息安 全、功能安全问题。信息安全分为两方面, 缺一不 可 [11]: 网络系统中各通信、计算机设备及相关设 施等有形物品都要得到保护, 即物理安全; 信息具 有完整性、保密性、可用性等, 即逻辑安全。功能 安全也分为两方面: 具有足够的安全功能, 对可能 发生的危险要有相应的安全防护措施; 每一种安全 功能都能得到可靠的执行, 无论在正常情况或者有 故障存在的情况下都应保证正确实施 [12]。

\section{（二）特种设备智能制造的发展要点}

\section{1. 产品可追溯}

充分利用现代信息技术, 建立特种设备产品追 溯体系, 实现产品及其质量安全的可追溯、可定位、 可管理, 这是特种设备产品智能化的重要方面, 也 是智能制造的重要内容。追溯体系采集记录产品生 产、流通、消费等环节信息, 实现来源可查、去向 可追、责任可究, 是强化全过程质量安全管理与风 险控制的有效措施, 在提升特种设备质量安全管理 能力、促进监管方式创新、保障使用安全等方面作 用显著。影响特种设备质量安全的环节多、链条长, 包括特种设备生产过程中的设计、工艺、检测、安 装, 使用过程中的操作、检查、维护、保养、检验 等; 由于质量安全信息追溯难度较高, 应充分利用 编码技术、先进传感、物联网、云计算、现代数据
库、大数据等, 构建特种设备智能化追溯体系, 实 现产品全过程质量安全跟踪与溯源, 全面提高信息 采集记录的快捷性、便利性、真实性、全面性、持 久性。

2. 质量管理智能化

质量管理是特种设备制造活动的重要内容, 也 是特种设备质量和安全的必要保障。我国特种设备 质量管理已形成 “法人管理、企业自检、第三方监 督、政府监察” 的体系模式, 但这一传统模式存在 一些难以克服的问题。一方面, 无论是企业自检还 是第三方监督、政府监察, 质量管理工作的主要形 式为随机抽取产品的少量、局部、部分环节进行检 测、检查、试验, 无法实现质量管理在时间与空间 上的全覆盖; 另一方面, 过分依赖于人的作用和影 响力, 而人在做事过程中存在随意性差异性并受精 神力量影响, 一旦相关人员责任心不强、心理状态 不稳定, 就可能影响检测、检查的结果, 进而影响 产品的质量与安全性能。

现代信息技术的发展为特种设备传统质量管理 模式的转型升级奠定了坚实基础, 宜利用先进传感、 AI、物联网、大数据等技术来构建智能化的质量管 理体系。(1)在特种设备设计质量管理方面, 传统设 计工作主要依靠人工或单机版的设计软件完成风险 评估、设计计算、输出设计文件等, 设计质量管理 手段主要包括设计人员（设计、审核、审批）资格 审查、设计依据的合规性审查、设计输出文件内容 的抽查等, 这些过程对质量管理人员的劳力、智力 要求高, 且对设计结果难以做到全覆盖验证; 智能 制造可以面向产品全生命周期, 具有丰富的设计知 识库、基于模拟仿真技术支持的智能化设计系统, 可在网络环境中并行、协同工作, 考虑失效模式更 全面、更具经验性并可模拟真实情况, 既能保证设 计质量, 又可以实现对设计结果的全面验证。(2)在 材料与零部件方面, 传统特种设备质量管理的控制 要素主要包括供应商资格审查、质量证明文件确认、 质量抽查复验等; 在智能制造模式下, 搭建采购商 与供应商间的联网互动平台, 采购商通过平台可以 全面掌握采购材料与零部件的质量, 材料供应商协 同完成制造过程, 这就保持了原材料质量的全生命 周期受控。(3)在工艺方面, 传统的特种设备制造工 艺纪律执行情况主要依赖人工方式进行抽查; 在智 能制造模式下, 利用计算机视觉、大数据分析等技 
术, 开发智能化的施工质量监控系统, 对工艺纪律 执行情况进行全面地监测、预警、纠偏。(4)在检验 试验方面, 开发智能检测系统可大幅提高检测效率, 实现精准检测。

\section{3. 动态风险实时监控}

特种设备智能制造，应重点考虑利用现代信息 技术，实现对设备动态风险的实时监测和智能化防 控。文献 [5] 以移动式承压设备为例, 提出了构建 全生命周期全链条大数据平台的思路, 汇聚设备质 量、使用、运行、检验、故障、损伤等方面的信息。 通过构建相应模型进行数据挖掘，对各类风险实施 实时监测, 不仅可对单体设备的失效风险进行监测, 还可以不同时间尺度（周期、时段）、不同空间范 围（区域、单位、行业）、不同应用领域视角（政 府监管、技术检验、经济发展、社会治理）的宏观 安全风险进行实时监测; 将风险分析结果提供给政 府监管部门、技术检验机构等，用于支持相应业务 工作。在风险达到一定程度、可能出现危险情况的 状态下, 可以进行预警, 根据具体需要设计相应预 警指标。基于全生命周期全链条大数据平台，可实 时或定期进行预警分析，对质量、安全、管理等方 面的规律性、趋势性、苗头性问题做到尽早发现、 及时向相关单位警示; 设备一旦发生事故，实施 快速应急响应, 对设备身份及质量、安全、管理 等方面的历史信息进行追溯, 为进一步的应急处 置和事故调查提供依据。在设备上加装智能终端, 自动采集事故特征参量数据并上传至大数据平台, 通过分析计算和专家辅助决策系统来自动生成应 急预案, 指导人员疏散、逃生, 并启动安全防护 等应急措施。

\section{四、基于智能网联的特种设备智能制造}

\section{（一）智能网联的内涵}

智能网联最早应用于汽车行业 [13], 是实现智 能汽车、智能交通的公认技术途径。本文将智能网 联的技术理念引入到特种设备行业 [4], 结合特种 设备的特点, 对智能网联内涵作进一步阐述。

\section{1. 智能}

智能的原意是用来形容人类具有的智慧和能 力。人类作为高级动物, 有感觉器官 (眼、耳、皮肤、 鼻、舌)、感觉 (视觉、听觉、触觉、嗅觉、味觉)、
神经、大脑、手、脚、肌肉等; 通过感觉器官感受 外界的光、声、热等信号, 通过神经将信号传送至 大脑, 大脑对信号进行分析判断后发出反馈信号, 最后指挥手、脚、肌肉等作出相应的反应。机器设 备的智能化指具有类似于人类的上述能力, 分为智 能感知识别、智能分析决策、智能控制执行、智能 记忆学习四方面。

智能感知识别指对自身状态与环境影响具有感 觉识别能力。利用传感器监测技术 (压力、温度、 物位、流量等传统监测技术，图像识别、语音识别 等 $\mathrm{AI}$ 技术)、物联网监控技术、互联网信息监测技 术、社交网络信息监测技术, 配置以高可靠的人工 定期检查, 对设备的工作参数、运行状态、环境条 件、使用情况、社会與论等方面信息进行实时采集、 搜索、分析并上传汇集, 支持进一步的智能分析和 决策。对特种设备来说，应重点实现对故障、损伤、 失效、风险相关的安全状态参量的感知识别。

智能分析决策指具有基于感知信息进行自行分 析判断和决策的能力。智能分析的内容包括对单台 设备的质量水平、安全状态、使用管理情况的分析, 对企业、机构、地区、行业的质量、管理、安全整 体状况等方面的分析, 根据分析结果作出停机、停 用、维保、检验、监督检查、报废等决策。分析决 策依赖丰富的模型算法和强大的知识库，建立基于 大数据的分析决策系统, 汇集全生命周期全链条的 大数据, 构建各类分析、诊断、评价、评估、预测、 预警模型, 然后运用大数据挖掘、云计算、现代统 计学等工具进行相应分析并作出相应决策。

智能控制执行指具有对决策指令进行自动响应 的能力。基于安全需求, 监测到设备的故障、损伤、 失效、事故、违规操作、管理缺位等异常情况后, 采取相应措施及时进行处理; 开发远程自动控制系 统, 对超出范围的工艺参数及时进行调整, 对违规 操作行为及时进行纠正; 在外界环境出现危险情况 时及时启动安全防护，在出现事故时及时进行应急 处置, 尽可能避免事故发生或减小事故后果。

智能记忆学习指具有积累经验和学习成长的能 力。利用云存储、现代数据库等技术构建大数据平 台, 进行数据分析和挖掘, 利用已经积累的数据指 导完成相应的业务和服务工作；在业务和服务工作 中不断积累新的经验数据, 将这些新数据纳入数据 库; 如此不断循环，使汇集数据的数量和质量不断 
提高, 数据知识库不断丰富, 实现自我学习成长。

2. 网联

网联是将相关的设备、人员、机构、环境等互 联起来构成网络（以下称为智联网，见图 1), 设备、 人员、机构、环境是智联网中的信息节点。智联网 与各节点之间可以信息交互：设备可将信息上传至 智联网, 智联网也可将信息分发至设备。网联通过 物联网、移动互联网、局域网等现代信息技术来实 现, 智联网汇集了特种设备基于时间、空间等多维 度的大数据, 通过模型构建、大数据挖掘等技术进 行各种应用开发，最终使全网的设备具备感知识别、 分析决策、控制执行、记忆学习等功能, 进而实现 网联式智能。相较以单台设备为对象的智能化系统, 通过智联网感知的信息将更全面、更完善，不仅可 以感知到某一台设备的信息, 还可以感知到与该台 设备相关的所有其他设备以及人员、机构、环境、 管理方面的信息。基于全网数据进行的分析决策 更可靠、更科学，控制执行更及时、更到位，记 忆学习更深入、更高效。

\section{（二）基于智能网联的特种设备智能制造关键任务} 发展特种设备智能制造, 首要的是确定技术途 径, 将智能网联作为推进特种设备智能制造的技术 方案，具有技术、工程、经济上的可行性，涉及的 关键任务如下。

\section{1. 构建特种设备智联网}

构建智联网是实现特种设备智能网联的前提, 可采取逐步推进的策略，先对不同类别、品种的设 备分别构建智联网, 然后将各品种设备智联网进一 步互联，最终形成整个特种设备 “一张网”。

(1) 智能监控系统配置

对纳入智联网的每台设备分别配置一套监控系 统, 监测设备的操作、运行、使用、管理等情况, 执行分析决策的指令; 重点监测设备的故障、损伤、 失效、事故等安全状态参量, 自动采取相应的预警 预报、安全防护、应急处置等控制措施。不同品种 设备的结构、原理、使用环境、运行模式不同，需 要配置不同的监控系统。(1)智能监控系统的监控指 标需综合科学性、系统性、实用性、有效性、可行 性等原则来确定, 通过安全风险及其影响因素的识 别, 结合法规标准要求, 篮选、凝练出针对性和实 用性相结合的指标体系。(2)监测技术应充分利用光

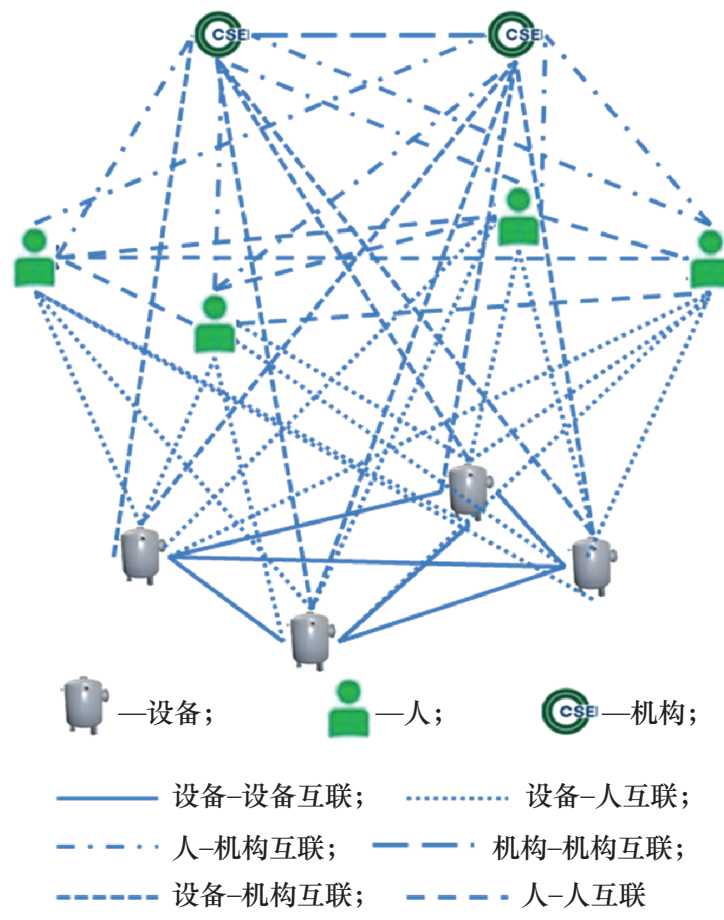

图 1 智联网示意图

纤光栅、振动、腐蚀探针、声发射、脉冲涡流、磁 记忆等传统技术, 雷达、超声波等远程传感技术, 语音、图像识别等 $\mathrm{AI}$ 技术，根据可靠性方面的特 殊要求开展进一步的专门研发。(3)通信模块是智能 网联特种设备的必要组成部分, 通信模块架构设计, 应对设备运行特点开展系统分析，综合考虑电源供 应、功耗、设备流动性、数据稳定性、入网设备数 量、数据流量等因素来选用。(4)控制执行系统既可 采用远程自动控制系统，也可人工采取技术或管理 手段进行可靠地干预。(5)监控系统应考虑功能安全 问题, 保证所监测到的数据真实可用; 数据存储和 传输应保密可靠, 控制执行系统在出现异常情况时 也能按照设计要求及时启动。

\section{(2) 搭建网联式数据平台}

智能网联特种设备需要以数据为支撑, 搭建网 联式数据平台, 汇集数据应尽可能全面, 避免出现 数据盲区。对于入网的设备, 应以其全生命周期全 链条各环节为线索, 以法规标准为基础, 对数据来 源、分布进行系统梳理, 理清数据流向、明确数据 总体架构; 建立数据目录体系，做好数据分类。

网联式数据平台的搭建需考虑以下因素。(1)平 台的总体架构设计应满足数据采集的便利性、动态 更新、海量存储等需求。(2)平台汇集的数据应规范, 
制定数据元、数据格式、信息分类编码等方面的标 准, 对实时采集的数据作统一要求, 对数据库的架 构以及数据处理、存储、转换、检索、导出、接入 等制定统一规范; 数据应具有可用性, 数据存储前 需要进行清洗、集成、转换、规约、䇻选、融合、 标准化、整合、装载等一系列处理, 提高数据质量, 匹配后续的数据挖掘处理。(3)数据的汇集方式, 除 通过监控系统实时采集外, 还可用人工录入、计算 机导入等方式。(4)充分考虑信息安全问题, 数据平 台及连接在平台中的设备, 其信息连接到网络中, 随时随地与外界发生联系，有可能被窃取、干扰甚 至修改; 基本要求是信息既能不被外界所影响, 又 不泄漏到外界。

\section{2. 智能应用开发}

特种设备智联网建立后, 可根据实际需要开发 各类智能应用，实现相关管理、服务的智能化，提 高工作效率和质量。除了开发智能化追溯系统、智 能化质量管理系统以外, 还可以基于进一步提升特 种设备安全水平的需要来开发以下智能应用。

(1) 智能诊断分析

诊断分析指对设备出现的故障、损伤进行预 报、分析和判断。基于网联式数据平台, 通过开发 并嵌入智能诊断分析模型，对设备进行实时诊断分 析, 自动识别设备的故障和损伤, 确定其部位、性 质、类别、程度、原因等, 指出故障和损伤发生发 展的趋势及后果，提出控制、消除故障和损伤的调 整、维修、治理措施。

(2) 动态风险评估

实时准确获知设备当前的风险状态（动态风 险), 是设备安全保障领域亟需解决的热点问题。 基于网联数据, 开发动态风险评估模型并嵌入智联 网, 对设备风险进行实时动态评估, 对各种故障、 损伤引起失效的可能性和后果进行分析计算, 提出 相应的防控措施, 实现对设备风险的自动识别、防 控与治理。

\section{（3）智能预警预报}

预警指在风险不可接受的情况或事件发生之 前, 向相关单位发出紧急信号、报告危险情况, 尽 量避免危害在不知情或准备不足的情况下发生, 最 大程度减轻危害所造成的损失。传统的预警技术根 据以往的规律总结或观测得到的可能性前兆提出预 警。基于网联式数据平台, 构建智能预警技术体系,
确定预警指标的阈值，建立预警系统和相应的预警 工作机制; 开发相应的模型, 如在网联式数据平台 基础上开发预警预报模块，实现实时自动预警预报。

（4）智能应急响应

在网联式数据平台基础上, 开发智能应急模块, 设备一旦发生事故，可实现快速应急响应；快速实 现对设备身份及质量、安全、管理等方面历史信息 的追溯, 为下一步开展应急处置和事故调查提供依 据。针对不同设备事故类型，构建相应的智能应急 处置终端, 实现对事故现场特征参量数据的自动侦 测, 为及时生成有针对性的应急预案提供数据支撑, 便于处置事故并减小事故后果。

\section{五、结语}

当前，特种设备在质量、安全方面遇到了发展 瓶颈, 迫切需要转型升级。第四次科技革命为特种 设备带来了新的发展机遇，对特种设备融合应用物 联网、大数据、 $\mathrm{AI}$ 等新一代信息技术, 发展特种设 备智能制造具有重要意义。本文研究分析了特种设 备的特点、发展现状及发展智能制造的需求, 引入 智能网联作为发展特种设备智能制造的技术路径, 分析了概念内涵，明确了发展特种设备智能制造 的策略和发展要点, 剖析了基于智能网联的特种 设备智能制造的关键任务。后续可据此开展深化 论证和探索实践, 更好推进特种设备领域的科技 攻关和应用工作。

\section{参考文献}

[1] 周济. 智能制造——中国制造2025”的主攻方向 [J]. 中国机械 工程, 2015, 26(17): 2273-2284.

Zhou J. Intelligent manufacturing: Main direction of "Made in China 2025" [J]. China Mechanical Engineering, 2015, 26(17): 2273-2284.

[2] 国务院. 中国制造 2025 [EB/OL]. (2015-05-08) [2019-10-08]. http://www.gov.cn/zhengce/content/2015-05/19/content_9784. htm.

The State Council of the People's Republic of China. Made in China 2025 [EB/OL]. (2015-05-08) [2019-10-08]. http://www.gov. cn/zhengce/content/2015-05/19/content_9784.htm.

[3] 谭建荣, 刘达新, 刘振宇, 等. 从数字制造到智能制造的关键技 术途径研究 [J]. 中国工程科学, 2017, 19(3): 39-44.

Tan J R, Liu X D, Liu Z Y, et al. Research on key technical approaches for the transition from digital manufacturing to intelligent manufacturing [J]. Strategic Study of CAE, 2017, 19(3): $39-44$. 
４］国家市场监督管理总局. 市场监管总局关于2019年全国特种设 备安全状况的通告 [EB/OL]. (2020-04-16) [2020-11-01]. http:// www.samr.gov.cn/tzsbj/tzgg/zjwh/202004/t20200416_314295. html.

State Administration for Market Regulation. Circular of State Administration for Market Regulation on the safety situation of special equipment in China in 2019 [EB/OL]. (2020-04-16) [2020-11-01]. http://www.samr.gov.cn/tzsbj/tzgg/zjwh/202004/ t20200416_314295.html.

[5] 刘三江, 陈祖志, 黄强华, 等. 智能网联特种设备科技与标准化 需求分析一以移动式承压设备为例 [J]. 中国特种设备安全, 2019, 35(4): 5-12, 33.

Liu S J, Chen Z Z, Huang Q H, et al. Technology and standardization needs analysis for intelligent connected special equipment: Taking transportable pressure special equipment for example [J]. China Special Equipment Safety, 2019, 35(4): 5-12, 33.

[6] 刘三江, 陈祖志, 李光海. 智能网联特种设备监管模式分析以移动式承压设备为例 [J]. 中国特种设备安全, 2019, 35(10): 5-9.

Liu S J, Chen Z Z, Li G H. Initial analysis of supervision system for intelligent connected special equipment: Taking transportable pressure special equipment for example [J]. China Special Equipment Safety, 2019, 35(10): 5-9, 13.

[7] 刘三江, 陈祖志, 薄柯, 等. 智能网联移动式承压设备检验模式 分析一以长管拖车为例 [J]. 科技与产业, 2020, 20(3): 178182.

Liu S J, Chen Z, Bo K, et al. Initial analysis of inspection system for intelligent connected special equipment: Taking transportable pressure special equipment for example [J]. Science Technology and Industry, 2020, 20(3): 178-182.
[8] 沈功田, 贾国栋, 钱剑雄. 特种设备安全与节能2025科技发展战 略 [M]. 北京: 中国标准出版社, 2017.

Shen G T, Jia G D, Qian J X. Special equipment safety and energy saving 2025 science and technology development strategy [M]. Beijing: China Quality and Standards Publishing \& Media Co., Ltd., 2017.

[9] 刘三江, 陈祖志, 黄强华. 智能网联特种设备监管平台的构建一 以移动式承压设备为例 [J]. 中国特种设备安全, 2018, 34(8): 1-8.

Liu S J, Chen Z Z, Huang Q H. Construction of monitoring and management platform for intelligent connected transportable pressure special equipment: Taking transportable pressure special equipment for example [J]. China Special Equipment Safety, 2018, 34(8): 1-8.

[10] 沈烈初. 读《中国制造2025》与“德国工业4.0”后的思考 [J]. 铸 造纵横, 2016 (1): 5-8.

Shen L C. Thinking after reading "Made in China 2025" and “German Industry 4.0” [J]. Foundry Panorama, 2016 (1): 5-8.

[11] 徐茂智. 信息安全基础 [M]. 北京: 高等教育出版社, 2006.

Xu M Z. Information security foundation [M]. Beijing: Higher Education Press, 2006.

[12] 冯晓升, 史学玲. 功能安全一一种保障安全的新思路 [J]. 中国 仪器仪表, 2005 (10): 46-54.

Feng X S, Shi X L. Functional safety: A new way to ensure safety [J]. China Instrumentation, 2005 (10): 46-54.

[13] 李克强, 戴一凡, 李升波, 等. 智能网联汽车(ICV)技术的发展现 状及趋势 [J]. 汽车安全与节能学报, 2017, 8(1): 1-14.

Li K Q, Dai Y F, Li S B, et al. State-of-the-art and technical trends of intelligent and connected vehicles [J]. Journal of automotive Safety and Engergy, 2017, 8(1): 1-14. 